\title{
A Case of Serous Tubal Intraepithelial Carcinoma Diagnosed by Cytology during the Operation
}

\author{
Kenji Niwa1 ${ }^{*}$, Tsuneo Ishihara ${ }^{1}$, Yoko Ueda ${ }^{2}$, Motoki Takenaka ${ }^{2}$, Tomomi Shiga ${ }^{2}$, Sakae Mori $^{3}$, \\ Keigo Kuwabara ${ }^{3}$, Yoshio Yamaguchi' ${ }^{3}$, Takuji Tanaka ${ }^{4}$ \\ ${ }^{1}$ Department of Obstetrics \& Gynecology, Gujo City Hospital, Gujo City, Japan \\ ${ }^{2}$ Department of Obstetrics \& Gynecology, Gifu University School of Medicine, Gifu City, Japan \\ ${ }^{3}$ Section of Laboratory Medicine, Gujo City Hospital, Gujo City, Japan \\ ${ }^{4}$ Department of Diagnostic Pathology \& Research Center of Diagnostic Pathology, Gifu Municipal Hospital, Gifu City, Japan \\ Email: ^kniwa.gujo913@gmail.com
}

How to cite this paper: Niwa, K., Ishihara, T., Ueda, Y., Takenaka, M., Shiga, T., Mori, S., Kuwabara, K., Yamaguchi, Y. and Tanaka, T. (2018) A Case of Serous Tubal Intraepithelial Carcinoma Diagnosed by Cytology during the Operation. Open Journal of Pathology, 8, 69-77.

https://doi.org/10.4236/ojpathology.2018.83008

Received: May 8, 2018

Accepted: June 24, 2018

Published: June 27, 2018

Copyright $\odot 2018$ by authors and Scientific Research Publishing Inc. This work is licensed under the Creative Commons Attribution International License (CC BY 4.0).

http://creativecommons.org/licenses/by/4.0/

\section{c) (i) Open Access}

\begin{abstract}
We experienced a case of serous tubal intraepithelial carcinoma (STIC), which is the earliest morphologically recognizable precursor of pelvic high grade serous carcinoma, diagnosed by cytology during the operation. A 48-year-old Japanese woman visited to our department because of abnormal cytological result and the left adnexal mass on female cancer screening. Pre-operatively, she was diagnosed as cervical intraepithelial neoplasia (CIN) 3, adenomyosis, and the left adnexal cystic lesion with papillary growth. At laparotomy, the left adnexal tumor turned out to be cystic fallopian tube with small papillary growth on the inner surface, but abnormal findings were not present on bilateral ovaries. The cytology of the imprint smears from the papillary projection and ascitic fluid showed positive, suggesting serous adenocarcinoma. Histopathological examination of the lesion revealed the left fallopian tube lesion was STIC with immunohistochemically positive reactivity against p53. No metastases including disseminated lesions were noted. The patient received four courses of systemic chemotherapy, and had no recurrent signs 10 months after the operation.
\end{abstract}

\section{Keywords}

Serous Tubal Intraepithelial Carcinoma, Cytology, Immonohistochemistry, p53, Ki-67

\section{Introduction}

The incidence of the fallopian tube cancer is reported to account for approx- 
imate $0.3 \%$ of all malignant gynecologic tumors and to show increased tendency of serous carcinoma, including ovarian and peritoneal cancers [1]. Histopathologically dysplastic changes were first reported by Piek et al. [2] in a series of prophylactically removed fallopian tubes without macroscopic changes of women with a predisposition for ovarian cancer. The changes were accompanied by alterations in carcinogenesis-associated proteins, including $\mathrm{p} 53$. These precursor lesions were later called serous tubal intraepithelial carcinoma (STIC). The lesions are not only associated with tubal adenocarcinoma, but also high-grade serous ovarian cancer (HGSOC) [2] [3] [4] [5] [6]. In addition, endometrioid adenocarcinoma and clear cell carcinoma may occur by shedding of STIC developed in more proximal tubal tissue or from endometriosis [4]. Morphological characteristics of STIC include a proliferation of non-ciliated epithelium showing nuclear stratification, marked nuclear pleomorphism, prominent nucleoli, and mitotic figures [7] [8]. Immunohistochemically, aberrant p53 protein expression and an increased Ki-67 proliferation index in the lesion are observed [7] [8].

The pathogenesis of ovarian cancer is diverse, and the origin of HGSOC still remains controversial. Approximate 11.7\% - 15.3\% of women with ovarian cancer have a family history of cancer, of which the majority has an inherited mutation in breast cancer susceptibility gene (BRCA) 1 or 2 [9] [10]. The incidence of STIC has been reported to range from $0.6 \%$ to $7 \%$ in BRCA mutations carriers [11] [12]. The tubal cytology is reported to be useful for ovarian cancer screening and detection [13]. More than $70 \%$ of non-hereditary or sporadic ovarian carcinoma and HGSOC involve fallopian tube and STIC is the earliest form of HGSOC with recognizable morphology [7] [14]. In addition, inflammation of the fallopian tube, such as ovulation, menstrual cytokines or infection, has been hypothesized to be a cause of ovarian malignancies [15] [16]. However, a few studies on cytology of STIC have been reported [13] [17]. We report here a case of STIC diagnosed by cytology during the operation.

\section{Case Report}

A 48-year-old Japanese woman, gravida 2 para 2 with normal menstruation cycle, was referred to the Department of Obstetrics \& Gynecology, Gujo City Hospital due to abnormal cytological findings (high-grade squamous intraepithelial lesion) and left adnexal mass on her cancer screening test. Her biopsy under colposcopy (Figure 1(A)) showed cervical intraepithelial neoplasia (CIN) 3 (Figure 1(B)). Transvaginal ultrasound examination (Figure 2) and magnetic resonance imaging (MRI) also detected papillary growth in the left cystic adnexal lesion (Figure 3). There were no enlarged lymph nodes and disseminated lesions. Clinical laboratory data, including tumor markers (CA125, $14.9 \mathrm{U} / \mathrm{ml}$; CA19-9, $6.3 \mathrm{U} / \mathrm{ml}$; and CA72-4, $5.3 \mathrm{ng} / \mathrm{ml}$ ) were within normal limitations.

Under our working diagnosis of left ovarian cancer (stage IA or IC), the patient underwent laparotomy one month after her first visit. She desired to preserve her ovarian function because of having normal menstruation cycle, if the 

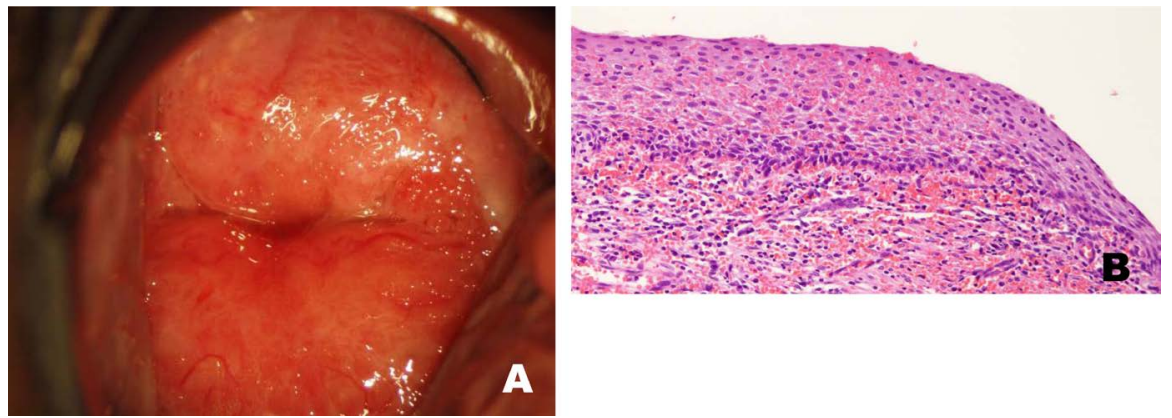

Figure 1. (A) Dense white epithelium is found in the colposcopy; (B) Histopathologic examination reveals CIN3 (H \& E stain, $\times 20$ ).

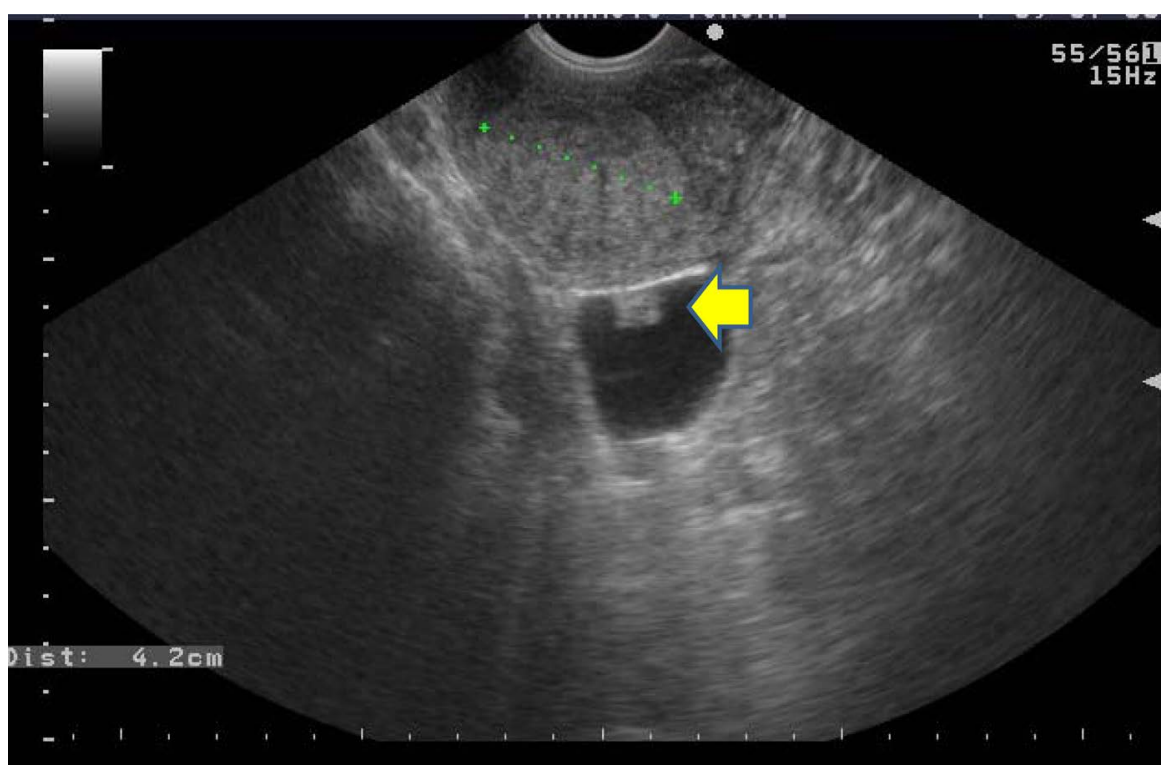

Figure 2. A transvaginal ultrasound examination shows a cystic lesion with papillary projections (yellow arrow) in the left adnexa, next to the uterus.

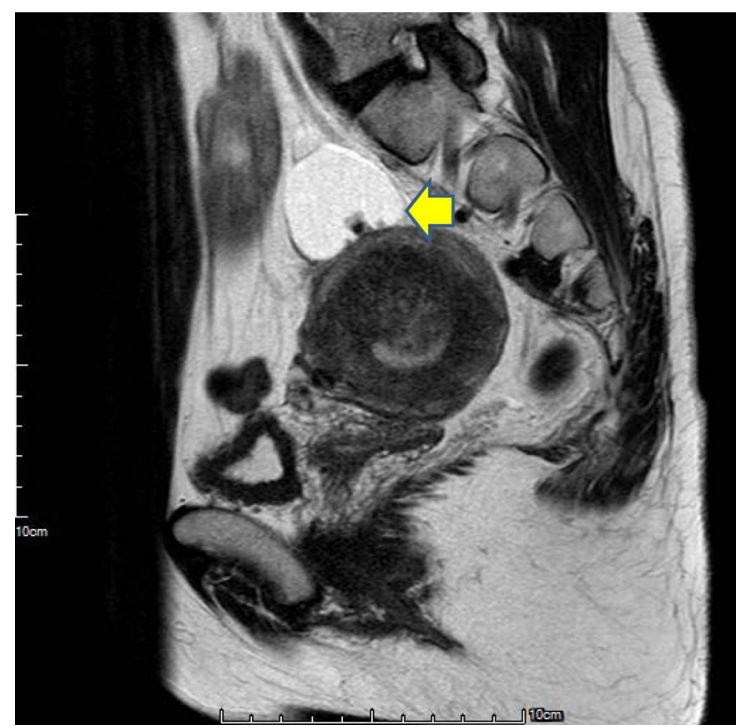

Figure 3. The MRI (T2-weighted) shows a cystic lesion with papillary projections (yellow arrow) next to the diffused enlarged uterus. 
tumor was benign. Before the operation, she was informed consents: she underwent a hysterectomy, left salpingo-oophorectomy, and right salpingotomy; if imprint cytology of the tumor showed positive, her right ovary must be removed; if ascites or washing cytology revealed positive, partial omentectomy and sampling of lymph node resection must be added. At the operation, approximate $5 \mathrm{ml}$ of slightly reddish ascites was sucked, and referred to the cytology. The tumor of left adnexa was cystic fallopian tube (Figure $4(\mathrm{~A})$ ), and papillary projections were noted in the inner surface (Figure $4(B)$ ), but abnormal findings could not been detected on the bilateral ovaries. The imprint smear of the projections (Figure 5(A)) and ascitic fluid cytology (Figure 5(B)) were positive, suggesting

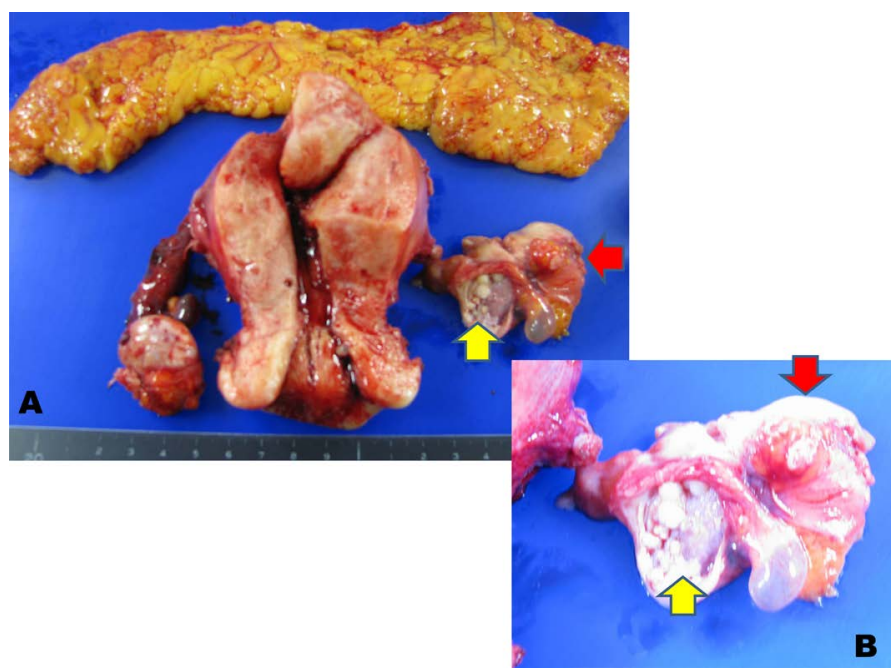

Figure 4. The left fallopian tube (red arrow) is enlarged and monocystic (A). Note: papillary projections in the inner surface (yellow arrow) (B). The ovaries show no significant abnormalities.

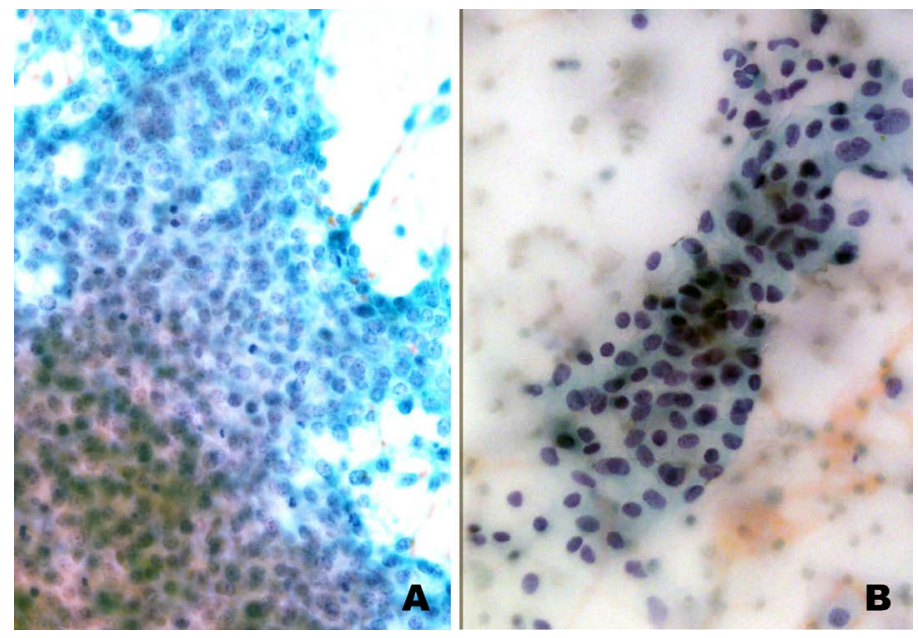

Figure 5. The imprint smear on the projections in the left fallopian tube (A) and cytology of the ascitic fluid (B). Both are positive, suggesting adenocarcinoma. Sheets of atypical cell cluster are noted in (A) and (B). The atypical cells with scarce cytoplasms exhibit hyperchromatic nuclei of variable sizes and irregular shapes, resulting serous adenocarcinoma. (A) and (B), Papanicolaou stain, $\times 100$. 
serous adenocarcinoma. Thus, partial omentectomy and sampling of lymph nodes resection were performed.

The resected fallopian cystic tumor was fixed in $10 \%$ buffered formalin, routinely processed, and embedded in paraffin. Three or four $\mu$ m-thick sections were stained with hematoxylin and eosin for pathological diagnosis. In addition, immunohistochemistry using Novocasta primary antibodies, such as p53 (1:30 dilution, Leica Biosystems, Tokyo, Japan) and Ki-67 (1:100 dilution, Leica Biosystems) was performed to determine the presence of p53 mutation and proliferative activity, respectively. Histopathological findings led to the diagnosis STIC in the left fallopian tube (Figure 6(A)) with strong positive reaction for p53 (Figure 6(B)) and 60\% positive rate of Ki-67 (Figure 6(C)), adenomyosis, and CIN3. Neither metastases nor disseminated lesions were found. The final diagnosis was left tubal STIC (pTis/1c3N0M0) with CIN3 and adenomyosis. From one month after the operation, she underwent four courses of systemic chemotherapy with paclitaxel $\left(175 \mathrm{mg} / \mathrm{m}^{2}\right.$ ) and carboplatin (AUC $6 \mathrm{mg} / \mathrm{ml} / \mathrm{min}$ every 21 days) [18] after fully informed consents that she wished to reduce her recurrent incidence and the ascitic fluid cytology was positive. She has no recurrent signs 10 months after the operation.

\section{Discussion}

Fallopian tube cancer is a very rare malignancy that is difficult to detect in the early stage. We described here a case of fallopian tube STIC diagnosed by cytology during the operation. In this case, we considered the left adnexal cystic tumor originating from the left ovary before operation. However, bilateral ovaries were free from tumor, and both the ascitic fluid cytology and imprint smears from the projection of the cystic tube showed positive during the operation. Based on the cytological findings, we added partial omentectomy and sampling of lymph nodes resection.

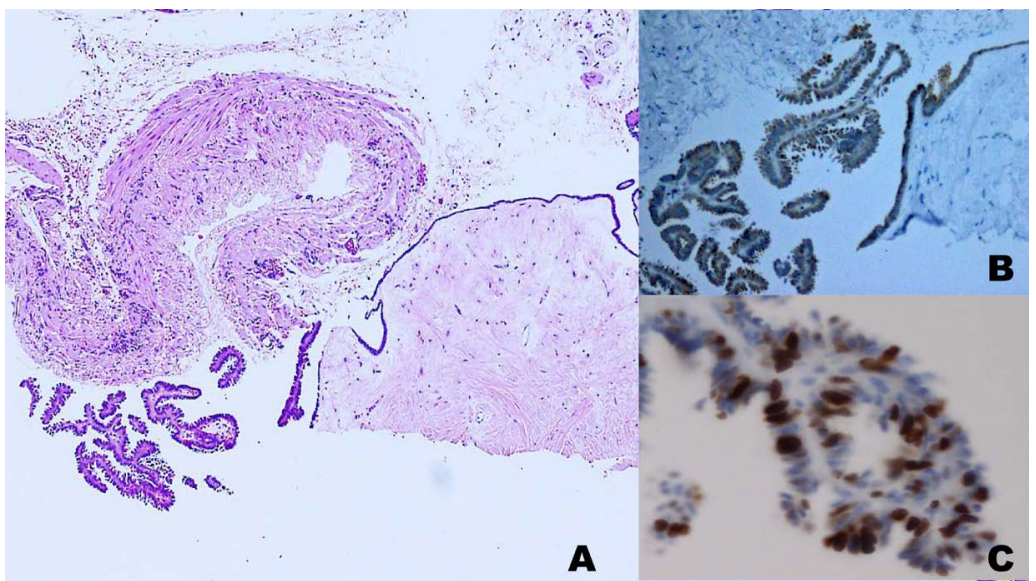

Figure 6. (A) Papillary growth of the atypical epithelium is present in the left fallopian tube ( $\mathrm{H} \& \mathrm{E}, \times 20$ ); (B) Immuhistochemistry of $\mathrm{p} 53$ shows strong positive reaction in the nuclei of the tumor cells $(\times 40)$; (C) Ki-67 immunohistochemistry revealed over $60 \%$ positive rate $(\times 80)$. 
STIC has been reported to occur in $0.6 \%$ to $7 \%$ women with BRCA1/2 mutations who receiving the prophylactic bilateral salpingo-oophorectomies [11] [12]. In our case, her past and family history related with breast or ovarian cancer could not be found, and the patient did not wish to examine the BRCA mutations. Since insurance coverage has not to be approved in Japan, the BRCA gene mutation analysis was not conducted.

Immunohistochemical examinations for p53 and Ki-67 were useful for the diagnosis of STIC in our case. The diagnosis of STIC is based on an assessment of both morphological and immunohistochemical features: the diagnosis is considered to be overexpression of p53 (over 60\%) and increased Ki-67 proliferative activity index [14] [19]. Immonohistochmeical features both HGSOC and STIC are reported to be clonally related, as identical p53 mutations occur in both STIC and concurrent HG serous carcinoma in the majority cases [20].

Fallopian tube tumors, including STIC, are rare in the gynecological malignant neoplasm [21]. Cytological diagnosis of this malignancy is difficult because of the technical problems, including sampling of fallopian tube epithelium. In general, this malignancy thus could be diagnosed by cervical [22] [23], vaginal [24], endometrial [25] or peritoneal [26] cytology. Cytology obtained by hydrotubation could be useful for diagnosing adenocarcinoma in situ of the fallopian tube [27]. Recently, an interesting cytological study on the fallopian tube during salpingo-oophorectomy has been reported by Chen et al. [13]. They have developed a standard procedure to collect fallopian tube brushing from freshly received surgical specimens and proved that tubal brushing cytology is useful for early detection of STIC and ovarian cancer screening [13].

The present case received postoperative adjuvant chemotherapy in accordance with the ovarian cancer treatment guideline, as the ascitic fluid cytology was positive. We chose four courses chemotherapy with paclitaxel and carboplatin. The relapse rate for administering chemotherapy in STIC with only positive ascitic fluid cytology is still unknown [18]. She has no recurrent signs 10 months after the operation, but we should conduct additional the long-term follow-up.

\section{Conclusion}

A case of STIC developed in a 48-year-old Japanese woman diagnosed by cytology during the operation was presented. Although pre-operative diagnosis was left ovarian cancer, the left adnexal tumor was found to be a cystic fallopian tube with papillary projections during the operation. The imprint smears of projections and ascitic fluid cytology were positive, suggesting serous adenocarcinoma. Histopathogical examination revealed that the lesion was STIC with p53 positive reaction. The patient underwent four courses of systemic chemotherapy, and has no recurrent signs 10 months after her operation.

\section{Acknowledgements}

We thank the editor and reviewers for the constructive comments, which helped 
us to improve the manuscript. This case report was approved by the patient verbal consent.

\section{Competing Interests}

The authors declare that they have no competing interests.

\section{References}

[1] Liao, C.I., Chow, S., Chen, L.M., Kapp, D.S., Mann, A., and Chan, J.K. (2018) Trends in the Incidence of Serous Fallopian Tube, Ovarian, and Peritoneal Cancer in the US. Gynecologic Oncology, 149, 318-323. https://doi.org/10.1016/j.ygyno.2018.01.030

[2] Piek, J.M., van Diest, P.J., Zweemer, R.P., Jansen, J.W., Poort-Keesom, R.J., Menko, F.H., Gille, J.J., Jongsma, A.P., Pals, G., Kenemans, P. and Verheijen, R.H. (2001) Dysplastic Changes in Prophylactically Removed Fallopian Tubes of Women Predisposed to Developing Ovarian Cancer. The Journal of Pathology, 195, 451-456. https://doi.org/10.1002/path.1000

[3] Kindelberger, D.W., Lee, Y., Miron, A., Hirsch, M.S., Feltmate, C., Medeiros, F., Callahan, M.J., Garner, E.O., Gordon, R.W., Birch, C., Berkowitz, R.S., Muto, M.G. and Crum, C.P. (2007) Intraepithelial Carcinoma of the Fimbria and Pelvic Serous Carcinoma: Evidence for a Causal Relationship. The American Journal of Surgical Pathology, 31, 161-169. https://doi.org/10.1097/01.pas.0000213335.40358.47

[4] Ottevanger, P.B. (2017) Ovarian Cancer Stem Cells More Questions than Answers. Seminars in Cancer Biology, 44, 67-71.

https://doi.org/10.1016/j.semcancer.2017.04.009

[5] Crum, C.P., Herfs, M., Ning, G., Bijron, J.G., Howitt, B.E., Jimenez, C.A., Hanamornroongruang, S., McKeon, F.D. and Xian, W. (2013) Through the Glass Darkly: Intraepithelial Neoplasia, Top-Down Differentiation, and the Road to Ovarian Cancer. The Journal of Pathology, 231, 402-412. https://doi.org/10.1002/path.4263

[6] Jarboe, E., Folkins, A., Nucci, M.R., Kindelberger, D., Drapkin, R., Miron, A., Lee, Y. and Crum, C.P. (2008) Serous Carcinogenesis in the Fallopian Tube: A Descriptive Classification. International Journal of Gynecological Pathology, 27, 1-9. https://doi.org/10.1097/pgp.0b013e31814b191f

[7] Kurman, R.J. and Shih Ie, M. (2010) The Origin and Pathogenesis of Epithelial Ovarian Cancer: A Proposed Unifying Theory. The American Journal of Surgical Pathology, 34, 433-443. https://doi.org/10.1097/PAS.0b013e3181cf3d79

[8] Visvanathan, K., Vang, R., Shaw, P., Gross, A., Soslow, R., Parkash, V., Shih Ie, M. and Kurman, R.J. (2011) Diagnosis of Serous Tubal Intraepithelial Carcinoma Based on Morphologic and Immunohistochemical Features: A Reproducibility Study. The American Journal of Surgical Pathology, 35, 1766-1775. https://doi.org/10.1097/PAS.0b013e31822f58bc

[9] Meyer, L.A., Anderson, M.E., Lacour, R.A., Suri, A., Daniels, M.S., Urbauer, D.L., Nogueras-Gonzalez, G.M., Schmeler, K.M., Gershenson, D.M. and Lu, K.H. (2010) Evaluating Women with Ovarian Cancer for BRCA1 and BRCA2 Mutations: Missed Opportunities. Gynecologic Oncology, 115, 945-952. https://doi.org/10.1097/AOG.0b013e3181da08d7

[10] Palma, M., Ristori, E., Ricevuto, E., Giannini, G. and Gulino, A. (2006) BRCA1 and BRCA2: The Genetic Testing and the Current Management Options for Mutation Carriers. Critical Reviews in Oncology/Hematology, 57, 1-23. 
https://doi.org/10.1016/j.critrevonc.2005.05.003

[11] Patrono, M.G., Iniesta, M.D., Malpica, A., Lu, K.H., Fernandez, R.O., Salvo, G. and Ramirez, P.T. (2015) Clinical Outcomes in Patients with Isolated Serous Tubal Intraepithelial Carcinoma (STIC): A Comprehensive Review. Gynecologic Oncology, 139, 568-572. https://doi.org/10.1016/j.ygyno.2015.09.018

[12] Zakhour, M., Danovitch, Y., Lester, J., Rimel, B.J., Walsh, C.S., Li, A.J., Karlan, B.Y. and Cass, I. (2016) Occult and Subsequent Cancer Incidence Following Risk-Reducing Surgery in BRCA Mutation Carriers. Gynecologic Oncology, 143, 231-235. https://doi.org/10.1016/j.ygyno.2016.08.336

[13] Chen, H., Klein, R., Arnold, S., Chambers, S. and Zheng, W. (2016) Cytologic Studies of the Fallopian Tube in Patients Undergoing Salpingo-Oophorectomy. Cancer Cell International, 16, 78. https://doi.org/10.1186/s12935-016-0354-x

[14] Mehra, K., Mehrad, M., Ning, G., Drapkin, R., McKeon, F.D., Xian, W. and Crum, C.P. (2011) STICS, SCOUTs and p53 Signatures; A New Language for Pelvic Serous Carcinogenesis. Frontiers in Bioscience (Elite Edition), 3, 625-634.

[15] Malmberg, K., Klynning, C., Floter-Radestad, A. and Carlson, J.W. (2016) Serous Tubal Intraepithelial Carcinoma, Chronic Fallopian Tube Injury, and Serous Carcinoma Development. Virchows Arch, 468, 707-713. https://doi.org/10.1007/s00428-016-1928-7

[16] Salvador, S., Gilks, B., Kobel, M., Huntsman, D., Rosen, B. and Miller, D. (2009) The Fallopian Tube: Primary Site of Most Pelvic High-Grade Serous Carcinomas. International Journal of Gynecological Cancer, 19, 58-64. https://doi.org/10.1111/IGC.0b013e318199009c

[17] Takeshita, R., Shoji, T., Mukaida, R., Sato, T., Sasou, S., Itamochi, H. and Sugiyama, T. (2017) A Case of High-Grade Serous Tubal Intraepithelial Carcinoma Diagnosed with Adenocarcinoma by Ascitic Fluid Cytology. Journal of Tumor, 5, 488-491.

[18] Bamias, A., Papadimitriou, C., Efstathiou, E., Rodolakis, A., Vlahos, G., Voulgaris, Z., Bozas, G., Fountzilas, G., Aravantinos, G., Razis, E., Gika, D. and Dimopoulos, M.A. (2006) Four Cycles of Paclitaxel and Carboplatin as Adjuvant Treatment in Early-Stage Ovarian Cancer: A Six-Year Experience of the Hellenic Cooperative Oncology Group. BMC Cancer, 6, 228.

[19] Weinberger, V., Bednarikova, M., Cibula, D. and Zikan, M. (2016) Serous Tubal Intraepithelial Carcinoma (STIC)-Clinical Impact and Management. Expert Review of Anticancer Therapy, 16, 1311-1321. https://doi.org/10.1080/14737140.2016.1247699

[20] Tone, A.A., Virtanen, C., Shaw, P. and Brown, T.J. (2012) Prolonged Postovulatory Proinflammatory Signaling in the Fallopian Tube Epithelium May Be Mediated through a BRCA1/DAB2 Axis. Clinical Cancer Research, 18, 4334-4344. https://doi.org/10.1158/1078-0432.CCR-12-0199

[21] Crum, C.P., Parkash, V., Alvarado-Cabrero, I., Piek, J.M.J., Bijron, J.G., Shaw, P., Carcangiu, M.L., Soslow, R., Ferry, J.A., van Diest, P.J. and Vang, R. (2014) Epithelial Tumours and Cysts. In: Kurman, R.J., Carcangiu, M.L., Herrington, C.S. and Young, R.H., Eds., WHO Classification of Tumours of Female Reproductive Organs, International Agency for Research on Cancer (IARC), Lyon, 103-112.

[22] Sasagawa, M., Nishino, K., Honma, S., Kodama, S. and Takahashi, T. (2003) Origin of Adenocarcinoma Cells Observed on Cervical Cytology. Acta Cytologica, 47, 410-414. https://doi.org/10.1159/000326542

[23] Hong, M.K., Lee, M.H., Ding, D.C., Chu, S.C. and Chu, T.Y. (2015) High Grade Serous Ovarian Carcinoma with Serous Tubal Intraepithelial Carcinoma in a Case 
Presented with Atypical Glandular Cell Favor Neoplasm Cervical Cytology and Dermatomyositis. Taiwanese Journal of Obstetrics \& Gynecology, 54, 183-186. https://doi.org/10.1016/j.tjog.2013.07.004

[24] Punnonen, R., Lauslahti, K. and Pystynen, P. (1985) Primary Malignancies of the Fallopian Tube. Annales Chirurgiae et Gynaecologiae. Supplementum, 197, 15-18.

[25] Maeda, D., Takazawa, Y., Ota, S., Takeuchi, Y., Seta, A., Nakagawa, S., Yano, T., Taketani, Y. and Fukayama, M. (2010) Bilateral Microscopic Adenocarcinoma of the Fallopian Tubes Detected by an Endometrial Cytologic Smear. International Journal of Gynecological Pathology, 29, 273-277. https://doi.org/10.1097/PGP.0b013e3181c30301

[26] Manchanda, R., Drapkin, R., Jacobs, I. and Menon, U. (2012) The Role of Peritoneal Cytology at Risk-Reducing Salpingo-Oophorectomy (RRSO) in Women at Increased Risk of Familial Ovarian/Tubal Cancer. Gynecologic Oncology, 124, 185-191. https://doi.org/10.1016/j.ygyno.2011.10.017

[27] Takahashi, M., Kigawa, J., Ishihara, K., Shimada, M., Kamei, T. and Terakawa, N. (2002) Hydrotubation for Diagnosing Carcinoma in Situ of the Fallopian Tube. A Case Report. Acta Cytologica, 46, 735-737. https://doi.org/10.1159/000326986 